$$
\text { CONF-970396--2 }
$$

\title{
Low Cost, Single Crystal-like Substrates For Practical, High Efficiency Solar Cells
}

\author{
A. Goyal ${ }^{1}$, D. P. Norton ${ }^{2}$, M. Paranthaman ${ }^{3}$, E. D. Specht ${ }^{1}$, Q. $\mathrm{He}^{2}$, \\ F. A. List ${ }^{1}$, D. M. Kroeger ${ }^{1}$, D. K. Christen ${ }^{2}$ and J. D. Budai ${ }^{2}$ \\ 'Metals \& Ceramics Division, ${ }^{2}$ Solid State Division. ${ }^{3}$ Chemical and Analytical Sciences Division, \\ Oak Ridge National Lab, Oak Ridge, TN 37831-6116.
}

\begin{abstract}
It is well established that high efficiency (20\%) solar cells can be routinely fabricated using single crystal photovoitaic (PV) materials with low defect densities. Polycrystalline materials with small grain sizes and no crystallographic texture typically result in reduced efficiences. This has been ascribed primarily to the presence of grain boundaries and their effect on recombination processes. Furthermore, lack of crystallographic texture can result in a large variation in dopant concentrations which critically control the electronic properties of the material. Hence in order to reproducibly fabricate high efficiency solar cells a method which results in near single crystal material is desirable. Bulk single crystal growth of PV materials is cumbersome, expensive and difficult to scale up. We present here a possible route to achieve this if epitaxial growth of photovoltaic materials on rolling-assisted-biaxially-textured-substrates (RABiTS) can be achieved. The RABiTS process uses well-established, industrially scaleable, thermomechanical processing to produce a biaxially textured or single-crystal-like metal substrate with large grains $(50-100 \mu \mathrm{m})$. This is followed by epitaxial growth of suitable buffer layers to yield chemically and structurally compatible surfaces for epitaxial growth of device materials. Using the RABiTS process it should be possible to economically fabricate single-crystal-like substrates of desired sizes. Epitaxial growth of photovoltaic devices on such substrates presents a possible route to obtaining low-cost, high performance solar cells.
\end{abstract}

\section{INTRODUCTION}

Significant progress is being made in the photovoltaics industry towards large volume production. Currently, photovoltaics is a $\$ 1$ Billion dollar per year business worldwide with more than 20 percent annual growth (1). Further increase in market share of the photovoitaic industry will require significant reductions in cost compared to most other forms of electricity generation typical in the utility industry. Higher efficiency cells made using more automated manufacturing processes are expected to lead the way to cost reduction. As prices decrease, usage of photovoltaic cells will increase, specially because of the environmetal benefits offered by this technology compared to other forms of energy generation. Further technological innovations have the potential to cause the economic and commercial breakthrough necessary to lower prices to $\$ 0.10$ per kilowatt-hour by the year 2000 and possibly by $\$ 0.04$ per kilowatt-hour by 2020 . In a recent overview

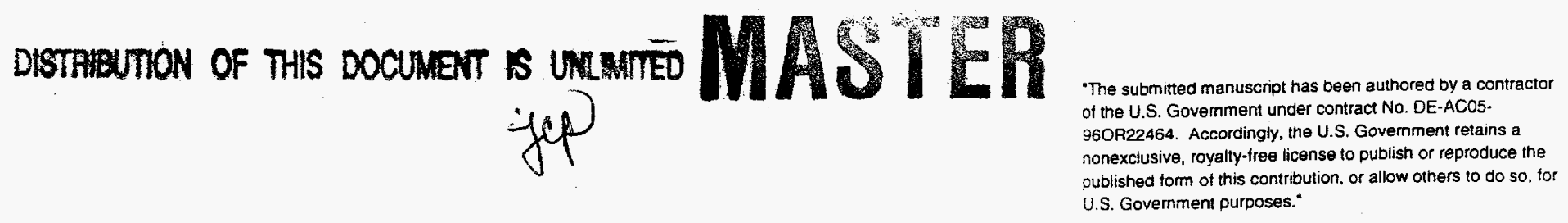




\section{DISCLAIMER}

This report was prepared as an account of work sponsored by an agency of the United States Government. Neither the United States Government nor any agency thereof, nor any of their employees, make any warranty, express or implied, or assumes any legal liability or responsibility for the accuracy, completeness, or usefulness of any information, apparatus, product, or process disclosed, or represents that its use would not infringe privately owned rights. Reference herein to any specific commercial product, process, or service by trade name, trademark, manufacturer, or otherwise does not necessarily constitute or imply its endorsement, recommendation, or favoring by the United States Government or any agency thereof. The views and opinions of authors expressed herein do not necessarily state or reflect those of the United States Government or any agency thereof. 


\section{DISCLAMMER}

Portions of this document may be illegible in electronic image products. Images are produced from the best available original document. 
article, Zweibel has pointed out that thin-film photovoltaics (PV) have a significant advantage over the traditional wafer-based crystalline Si cells (2). The primary advantage of thin films is cheaper materials and manufacturing costs and higher manufacturing yields compared to single-crystal technologies. Thin films use $1 / 20$ to $1 / 100$ of the material needed for crystalline Si PV and appear to be amenable to more automated, less expensive production (2). Thin-film PV modules have a very low projected potential cost (under $\$ 50 / \mathrm{m}^{2}$ ) and reasonable module efficiencies (13-15\% or more), implying potential module costs well under $\$ 0.5 / \mathrm{Wp}$. (2). Currently, three film technologies are receiving significant interest from the industry for large scale PV: amorphous $\mathrm{Si}, \mathrm{CuInSe}{ }_{2}$ and $\mathrm{CdTe}$. Module efficiencies are closely related to cell efficiencies, with minor losses $(-10 \%)$ due to some loss of active area and some electrical resistance losses (2). However, even today's best laboratory-level modules are about $8-10 \%$ efficient (2). When product-level technology and process development has adopted most technical capabilities observed in individual laboratory experiments, best laboratory modules are expected to be $-90 \%$ of efficiency of best cells. Furthermore, off-the-shelf commercial modules are expected to be about $90 \%$ as efficient as the best prototype modules (2). Based on the above considerations, a PV thin film technology that can produce cells with efficiencies in the range of $20 \%$ in a controllable and reproduceable manner is required. An efficiency of $17.7 \%$ using a thin-film, polycrystalline $\mathrm{CuInGaSe}$ (CIGS) has recently been demonstrated (1). In order to further increase the efficiency and to be able to reproduceably fabricate high effciency cells, further research is required to understand the microstructural features which limit the performance.

Current research at obtaining high efficiency cells is aimed at controlling the microstructural features of the film closely. While a complete understanding of the microstructral features which limit the performance are still unclear, it is reasonably well established that recombination at grain boundaries, intragrain defects and impurities is critical. In an effort to minimize the effect of grain boundaries, films with large grains are an objective. It has been estimated by Kurtz and McConnell that to achieve over $20 \%$ efficiency in GaAs solar cells, grain sizes close to 50-100 $\mu \mathrm{m}$ are required (3). This assumes grains with crystalline perfection less than $5 \mathrm{x}$ $10^{6} / \mathrm{cm}^{2}(3)$. While efficiencies close to this $(18-20 \%)$ have been recently attained by Venkatasubramanian et al. (4) in epitaxial GaAs films on cast, optical grade Ge substrates with grain sizes close to $1 \mathrm{~mm}$, the cost of raw optical grade Ge is high and it is not clear whether it can be reduced to an acceptable range for even the 1sun, terrestrial solar cells (3). Furthermore, since all thin-films discussed above are polycrystalline, they do not have a well-defined crystallographic orientation (both out-of-plane and in-plane). Crystallographic orientation can have two important effects. The first is the effect of orientation of the growth surface on incorporation of dopants, intrinsic defects, and other impurities (3). Studies on a wide variety of dopants have shown that variations of 1 to 2 orders of magnitude can occur based on crystallographic orientation $(5,6)$. An extreme effect of anisotropic doping is Si doping in GaAs films (3). Si doping in GaAs films, causes n-type conduction on (111)B-type GaAs, but p-type on (111)A-type GaAs (7). The second effect of crystallographic orientation is a variation in growth rate (3). Both experiments as well as simulations have shown that under certain 
conditions growth rates can vary by 1 to 2 orders of magnitude as a function of crystallographic orientation (8). Uncontrolled crystallographic orientation in PV materials with large grain sizes may therefore result in reproducibility problems and hence lower yields during high volume production.

Most of the microstructural features currently thought to be limiting polycrystalline, thin-film, solar cell performance can be avoided by growing epitaxial films on lattice-matched, single crystal substrates. However, the high costs of single crystal substrates prohibits their use for realistic applications. We present here a low cost substrate for potential growth of epitaxial PV films, which very closely resembles a single crystal crystallographically. The Rolling-assistedbiaxially-textured-substrates (RABiTS) technique has been recently proposed by the authors (19-21). The technique uses well established, industrially scaleable, thermomechanical processes to impart a strong biaxial texture to a base metal. This is followed by vapor deposition of epitaxial buffer layers (metal and/or ceramic) to yield chemically and structurally compatible surfaces. Epitaxial superconductor films grown on such substrates have critical current densities approaching $10^{6}$ $\mathrm{A} / \mathrm{cm}^{2}$ at $77 \mathrm{~K}$ in zero-field and have a field dependence similar to epitaxial films on single crystal ceramic substrates. Deposited conductors made using this technique offer a potential route for the fabrication of next generation high temperature superconducting wire, capable of carrying very high currents in high magnetic fields and at elevated temperatures. The basic substrate made by this technique can also be used for epitaxial deposition of elemental, II-VI, III-V, and I-III-VI semiconductors like Si, Ge, GaAs, ZnSe, ZnTe, CdTe and CIGS. We summarize here key microstructural features of substrates made using the RABiTS technique and how they could be used for epitaxial growth of semiconductors. As is illustrated in this paper, substrates made using this technique have large grains ( $>$ $100 \mu \mathrm{m})$ and primarily low angle grain boundaries.

The effect of grain boundaries can be circumvented in polycrystalline photovoltaic thin films if the grain sizes are large enough (grain size at which effects on properties are minimal depend among other things on the doping level). However in thin-films, grain growth is typically restricted to only twice the thickness of the film. Hence, grain boundaries in polycrystalline films have a dominant effect on efficiencies. A large number of studies have reported the effects of grain boundaries on photovoltaic properties. In order to establish the potential of biaxially oriented substrates, an overview of grain boundaries in semiconductors and their effects on photovoltaic properties is first presented.

\section{GRAIN BOUNDARIES IN SEMICONDUCTORS AND THEIR EFFECTS ON PHOTOVOLTAIC PROPERTIES}

Grain boundary (GB) effects in semiconductors can be classified as intrinsic or extrinsic in nature. "Intrinsic" GB effects correspond directly to disruptions in lattice bonding with a lattice relaxation to accommodate the misfit at the GB. Lattice relaxations result in the generation of dislocations arrays at the GB plane and in the covalent diamond lattice are accompanied by dilated, contracted and in some cases dangling bonds. These bonding effects can result in electronic states in the 
semiconductor bandgap. Hence intrinsic effects which occur due to GBs are bond distortion and dangling bonds. Moreover, the one dimensional periodicity along the dislocation core can lead to banding of these states. "Extrinsic" GB effects occur due to the interaction of the boundary with other lattice defects such as point, line and other planar defects. Furthermore, GB segregation and diffusion can result in variations of impurities or dopant atom concentrations at the GB relative to the bulk. This can have a direct effect on the chemical potential (Fermi level) associated with the boundary region. This in tum can result in localized electronic states in the bandgap and also alter the transport properties in the vicinity of the GB due to nonuniform dopant or impurity concentrations. Electronic states in the bandgap created due to such intrinsic and extrinsic defects can act as charge carrier traps and recombination centers and result in band bending, reduced carrier lifetime, and leakage currents associated with the GB.

Although many studies probing the effect of grain boundaries in various semiconductors have been made; no unified theory applicable to all types of materials exists (9-18). Some of the measured effects of grain boundaries are summarized here. Much of the early GB studies focussed on Ge. It has been established that the GB behaves as though it contained acceptor-like electronic states in the bandgap giving rise to band bending in such a fashion so as to create a barrier to minority carrier transport across GBs in n-type Ge. These states were also found to create a hole-like conductivity in the GB plane that is independent of temperature. The structure of the GB as predicted by the crystallography has been shown to dictate the bonding structure and hence influence the properties. In contrast to Ge, GB potential barriers have been found to occur in both n-type and p-type Si. This may be due to pinning of the Fermi level by the GB states at different positions or the ability of these states to act both as electron and hole traps. Detailed transport studies have shown how GB states give rise to the back-to-back diode characteristic for thermionic current flow across a grain boundary. However, not all grain boundaries were found to be electrically active. For example, it has been observed that high-angle coherent twin boundaries are electrically inactive. Twin boundaries have a high degree of coincidence and hence a lower energy than other high-angle boundaries. This points to the importance of GB energy, its effect on GB structure and resulting electronic properties. Enhanced impurity diffusion along GBs has also observed in Si and Ge. Low-angle GBs in both $\mathrm{Si}$ and $\mathrm{Ge}$ have been shown to be composed of discrete dislocations $(11,12)$. This description is valid for [110] tilt boundaries with misorientation angles of less than $5^{\circ}(11)$. For twist GBs this model fails for misorientation angles in the range of $3^{\circ}$ to $8^{\circ}(12)$. A change in the GB structure that occurs with the transition from low-angle to large misorientations is indicated by the electronic properties of Ge GBs. For example, the anisotropic nature of transport in the grain boundary plane, indicative of the presence of discrete dislocations disappeared as the misorientation angle increased (13). In another study, tilt and twist GBs, both having a misorientation angle of $6^{\circ}$, displayed the same properties although their dislocation structures should be different (14). This is consistent with the transition from low angle regime observed to occur at approximately $5^{\circ}(11,12)$. In some high angle boundaries, microfacets are known to form leading to GB plane orientations that contain a high planar density of coincident lattice sites (CSL). This arrangement is achieved by a 
relative translation of one grain with respect to another and can be physically interpreted as the reconstruction of the bonding structure at the interface resulting in the formation of five, six, and seven member rings at the GB (15). No translation is associated with the coherent twin boundary with an exact coincident structure (15).

GB potential barriers have also been shown to exist in both n-type and p-type GaAs, the barrier however is significantly lower in n-type material. An experimental study by Salermo et al. (9) studied the characteristics of bicrystal prepared using the lateral overgrowth technique. They studied n-type GaAs bicrystals containing [110] GBs with misorientations of $0^{\circ}, 2.5^{\circ}, 5^{\circ}, 10^{\circ}, 24^{\circ}$ and $30^{\circ}$. Current-voltage, capacitance-voltage and deep level transient spectroscopy (DLTS) were used for electrical characterization of the GBs. It was found that the rectification associated with tilt GBs increased as a function of misorientation angle. For GBs greater than $10^{\circ}$, the potential barrier height was found to remain constant. Moreover, the slight rectification associated with the $2.5^{\circ}$ and $5^{\circ} \mathrm{GBs}$ indicates that the donor density of these layers is high enough to essentially the GB trap states.

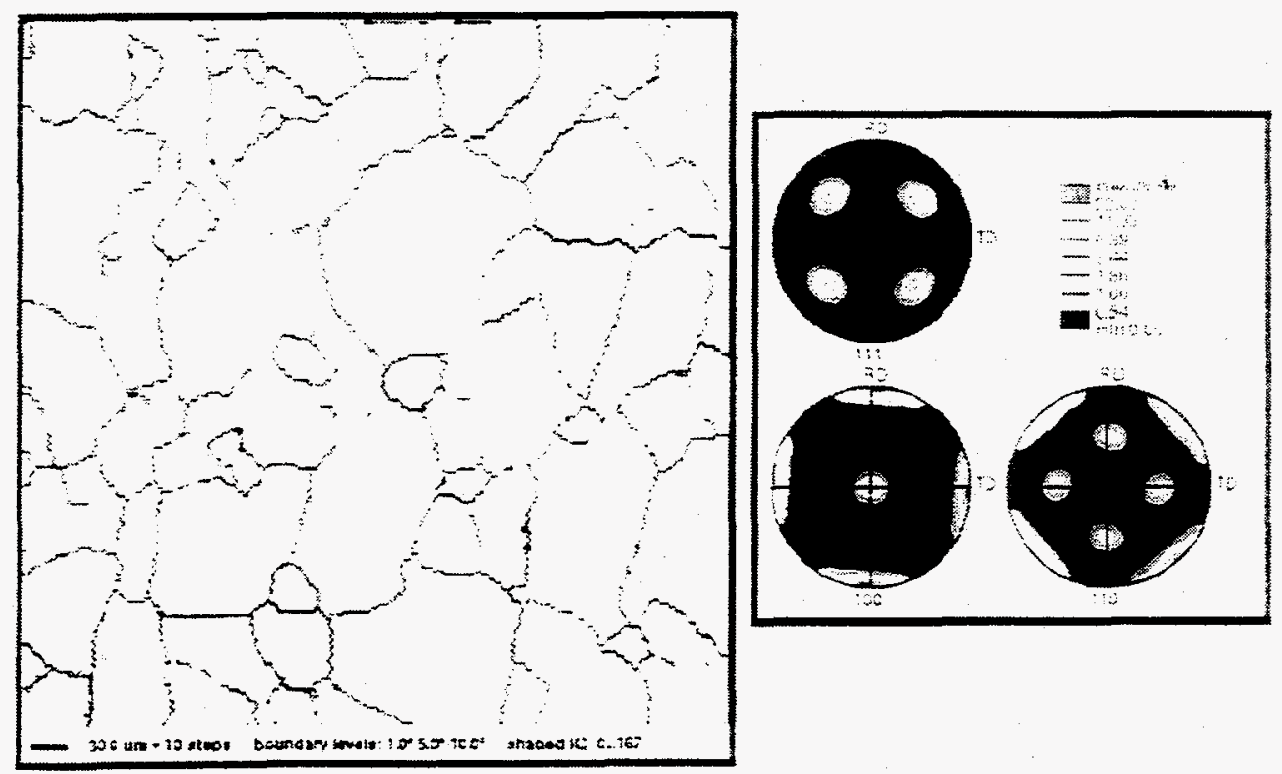

FIGURE 1. (a) Orientation image micrograph from a macroscopic region of a RABiT substrate (see text for details). BKD patterns were obtained in a hexagonal grid with a step size of $3 \mu \mathrm{m}$. Variations in intensity are a reflection of pattery quality or intensity of the Kikuchi bands. Three types of GBs are indicated in the figure, thin GBs denote boundaries with misorientations greater than $1^{\circ}$ and less than $5^{\circ}$, thicker lines denote GBs with misorientations greater than $5^{\circ}$ and less than $10^{\circ}$ and the thickest boundaries denote boundaries with misorientations greater than $10^{\circ}$; (b) (111), (100), (110) pole figures of the orientations used to construct Figure 1 (a). Only a single orientation, $\{100\}<001>$ cube texture is present. 
Furthermore, the breakdown or saturation voltage was found to be a strong function of misorientation angle. These data indicate that the density of GB states increases with misorientation angle until saturation occurs in the range of $24^{\circ}$ to $30^{\circ}$, suggesting that GB states in GaAs are directly related to GB structure.

GB effects on device characteristics can be described by considering the two cases of current flow and parallel and perpendicular to the boundary. For most photovoltaic materials, bulk as well as thin films, the grain structure is columnar with the grain boundary plane approximately normal to the junction, thus the case of current flow parallel to the GBs is of interest. Typically the polycrystalline device is modelled as both bulk and GB p-n junctions in parallel, with the bulk and $\mathrm{GB}$ regions as having different bandgaps and lifetimes. For current flow parallel to the boundary, GBs act as high conduction paths and lower the open circuit voltage and fill factor of the device. For current flow perpendicular to the GB, band bending at the boundary results in a potential barrier and therefore majority carrier transport across the GB encounters high resistance. This can result in increase in the series resistance of the device and hence :educe the short circuit current and the fill factor. Modelling of GB effects on properties has been performed by Card and Yang (16), who systematically developed the dependence of minority carrier lifetime, $\tau$, on doping concentration, $N_{d}$, grain size and interface density of states $\left(\mathrm{N}_{\mathrm{is}}\right)$ in Si. Judging from past work on heterojunctions, they postulated that the interface density of states can vary non-linearly by factors of $10^{2}$ to $10^{3}$ between low $\left(\sim 10^{10} \mathrm{~cm}^{2} \mathrm{eV}^{-1}\right)$ and high angle $\left(>10^{13} \mathrm{~cm}^{2} \mathrm{eV}^{-1}\right)$ GBs. Since minority carrier lifetime is inversely proportional to $\mathrm{N}_{\text {is }}$, a transition from low to high angle GB results in 3 to 4 orders of magnitude change in $\tau$. It was shown that for poly-Si, $\tau$
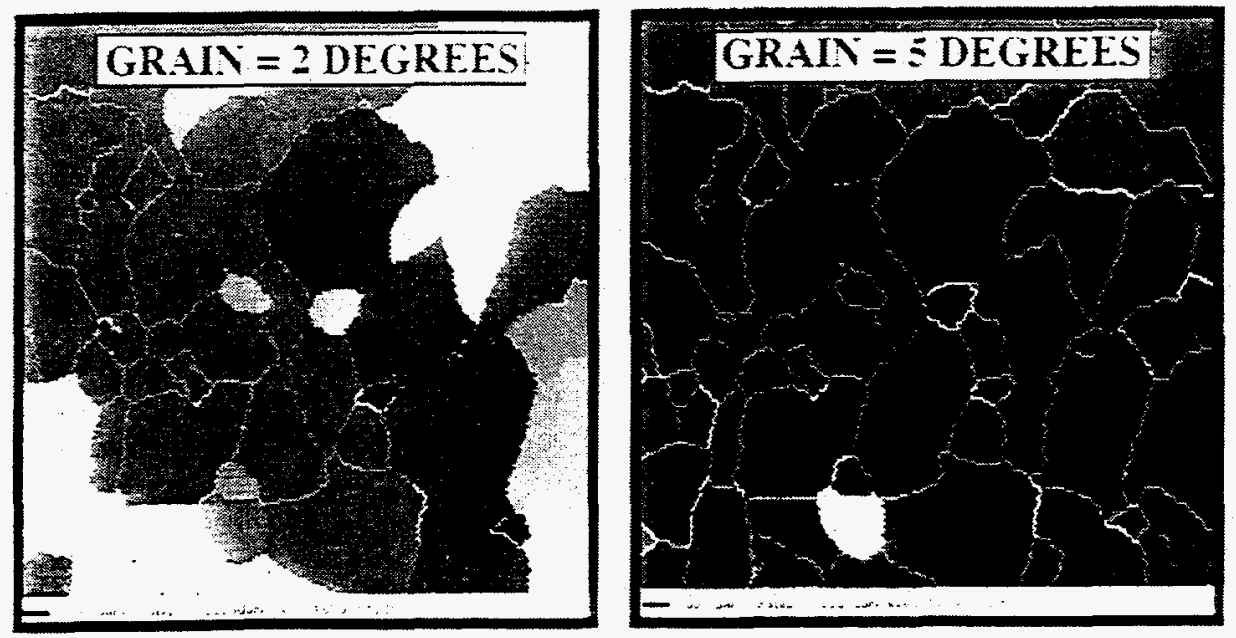

FIGURE 2. Orientation image micrographs shown in Figure 1a, shaded with the criterion that a given color represents a percolative region within (a) $2^{\circ}$ and (b) $5^{\circ}$. Clearly most of the substrate is well connected by boundaries less than $5^{\circ}$. 
varies by 4 orders of magnitude, from $10^{-6}$ to $10^{-10}$ as the grain size varied from 1000 to $0.1 \mu \mathrm{m}$ (for a constant $\mathrm{N}_{\mathrm{f}}$ ). For a constant grain size, $\tau$ varied by 2 orders of magnitude with $\mathrm{N}_{\mathrm{is}}$. Similar calculations have been extended to other materials including GaAs (17) and $\mathrm{CuInSe}_{2}$ (18).

The above discussion establishes the importance of controlling GB types in photovoltaic thin fims. Biaxially textured, columnar films with large effective grain sizes would be ideal. An effective grain size is one where grain boundaries within a certain misorientation can be tolerated. From the discussion above, it appears that for PV materials, this may be in the vicinity of $4-5^{\circ}$. Hence, substrates with GBs in this regime would be ideal. A second approach would be to have substrates with very large grains, so that grain boundary effects are minimal. Kurtz and McConnel have shown that for GaAs, this grain size is in the vicinity of $\sim 100 \mu \mathrm{m}$. The RABiTS technique combines both these approaches and achieves very large individual grains $(>100 \mu \mathrm{m})$, with primarily low angle grain boundaries less than $5^{\circ}$.

\section{ROLLING-ASSISTED-BIAXIALLY-TEXTURED- SUBSTRATES (RABITS)}

RABiTS is a technique to produce macroscopically biaxially textured substrates (19-21). The method employs thermomechanical processing of base metals such as $\mathrm{Cu}$ or $\mathrm{Ni}$ to obtain a very sharp, well developed $\{100\}<100\rangle$, cube texture. This is followed by deposition of appropriate chemical and structural buffer layers on the textured base metal. Substrates with biaxially textured, chemically and structurally compatible surfaces for epitaxial growth of superconducting or other electronic devices are referred to as rolling-assisted-biaxially-textured-substrates (RABiTS). Substrates made this way can be thought of as very large, defected, single crystals.

Biaxially textured $\mathrm{Ni}$ substrates were formed by consecutive rolling of a polycrystalline, randomly oriented high purity (99.99\%) bar to total deformations greater than $90 \%$ (19). By controlling the surface condition of the work rolls, it was possible to obtain substrates with surfaces as smooth as those obtained by

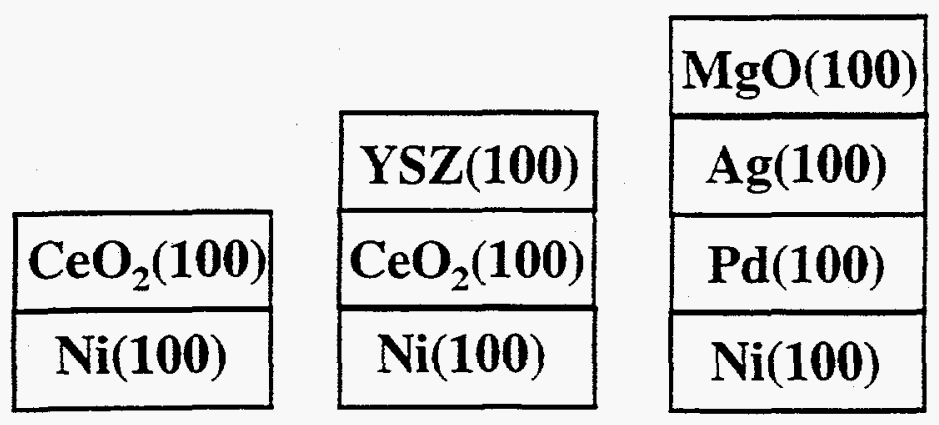

FIGURE 3. Schematic representations of cross-sections of three RABT multilayer structures, $\mathrm{CeO}_{2} / \mathrm{Ni} ; \mathrm{YSZ} / \mathrm{CeO}_{2} / \mathrm{Ni} ; \mathrm{MgO} / \mathrm{Ag} / \mathrm{Pd} / \mathrm{Ni}$. 


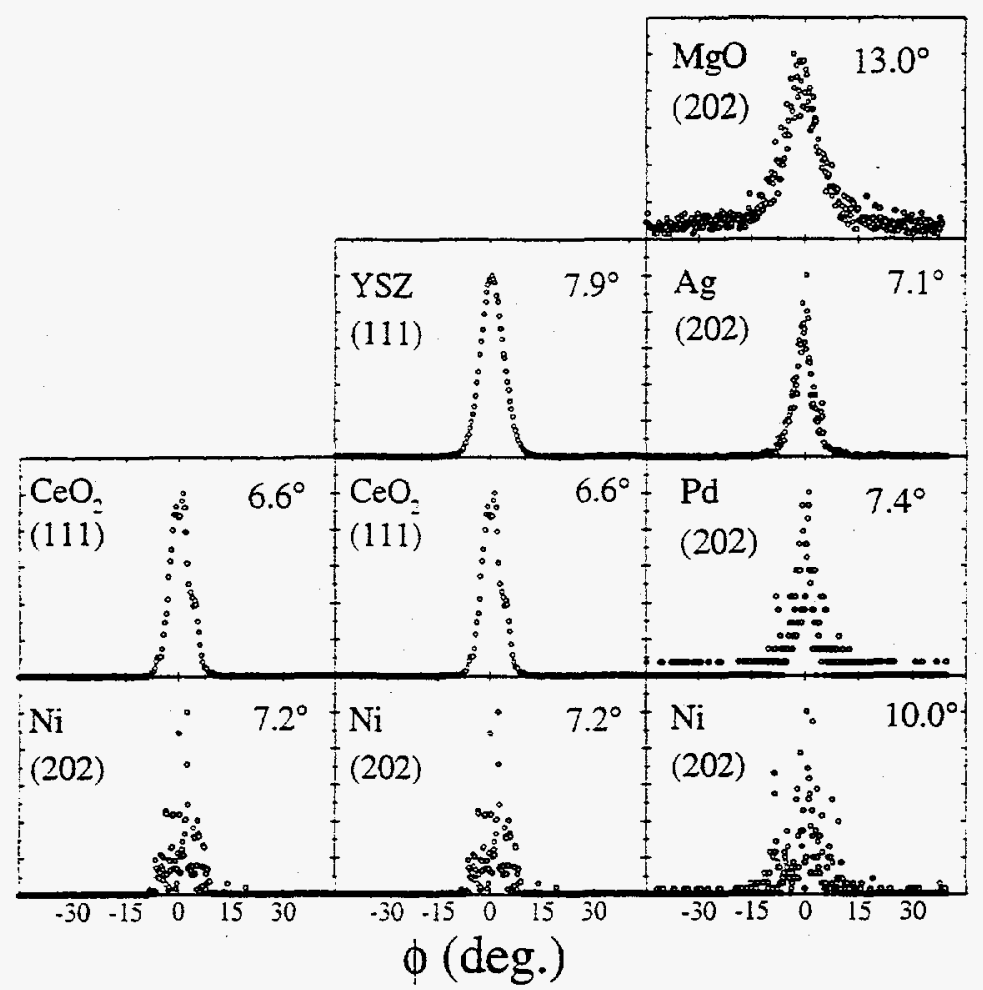

FIGURE 4. X-ray $\phi$-scans showing the transference of texture through the various multilayers of a RABIT substrate, starting from the rolled and recrystallized metal to the top oxide layer: a) $\mathrm{CeO}_{2}<110>\| \mathrm{Ni}<100>$; b) $\mathrm{YSZ}<110>\left\|\mathrm{CeO}_{2}<110>\right\| \mathrm{Ni}<100>$; and c) $\mathrm{MgO}<100>\|\mathrm{Ag}<100>\| \mathrm{Pd}<100>\| \mathrm{Ni}<100>$.

mechanical and chemical polishing, with rms roughnesses of $\sim 10 \mathrm{~nm}(21)$. The surface condition of a substrate can greatly affect epitaxy and integrity of buffer layers. Obtaining substrates with surfaces adequate for film growth without the need for a cumbersome polishing step is important for scale up to long lengths or large areas. Subsequent annealing of the substrates in a wide temperature range results in the formation of a sharp $\{100\}<100>$ cube texture. Figure 1a shows an orientation image micrograph of a macroscopic region of a substrate recrystallized at $1000^{\circ} \mathrm{C}$ for $4 \mathrm{hrs}$ in a vacuum of $\sim 10^{-6} \mathrm{Torr}$. The micrograph was obtained using electron backscatter kikuchi diffraction (BKD). Gray level shading on the micrograph is a reflection of the pattern quality or intensity of the kikuchi bands observed at each point. Grain boundaries give rise to multiple diffraction patterns and hence have a poor pattern. Similarly, poor patterns are observed from any other crystallographic defect or strained region. BKD pattems were obtained on a hexagonal grid with a spacing of $3 \mu \mathrm{m}$. Total number of patterns obtained in the $0.5 \mathrm{~mm} \times 0.5 \mathrm{~mm}$ region were close to 30,000 . Indexing of the pattern at each location gave a unique measure of the orientation at that point. A hypothetical hexagonal lattice with a grain size of $3 \mu \mathrm{m}$ was superimposed at each point from where a pattern was obtained. Grain boundary misorientations were then calculated 
for all the resulting boundaries using standard techniques. The micrograph was then regenerated with certain grain boundary criteria. In Figure 1a, three sets of GBs are indicated. The thin boundaries are boundaries with misorientation angles greater than $1^{\circ}$ and less than $5^{\circ}$. Thicker boundaries have misorientation angles greater than $5^{\circ}$ and less than $10^{\circ}$, and the thickest boundaries have misorientations greater than $10^{\circ}$. Regions where no boundary is indicated, but the contrast is dark correspond to boundaries with misorientations less than $1^{\circ}$. Figure $1 \mathrm{~b}$ shows (111), (200) and (110) pole figures constructed using the data from Figure la. The presence of a sharp, well-developed, single component cube texture is evident. Typical samples have $\mathrm{X}$-ray $\omega$ - and $\phi$-scans with full-width-half-maximum (FWHM) of $6^{\circ}$ and $7^{\circ}$ respectively. The texture is found to be stable up to the melting point of $\mathrm{Ni}$. Figures $2 \mathrm{a}$ and $\mathrm{b}$ show coloring of the same region as shown in Figure 1a, according to a criterion that a single color represents a contiguous or percolative region of orientation less than $2^{\circ}$ and $5^{\circ}$ in Figure $2 \mathrm{a}$ and $\mathrm{b}$ respectively. It can be seen that mosi of the substrate is percolatively connected within $5^{\circ}$. After recrystallization, the average grain size is approximately equal to the thickness of the substrate, which in this case was $125 \mu \mathrm{m}$. Thus the substrate can be thought to be comprised of a columnar structure of grains, with the columns aligned with the (100) plane parallel to the surface of the columns and the [100] direction aligned along the rolling direction. Typically, it is expected that for metal sheets with columnar grains, grain growth saturates at approximately twice the thickness of the substrate. In this case, the highly reduced mobility of low angle boundaries appears to saturate the grain size to approximately that of the substrate thickness.

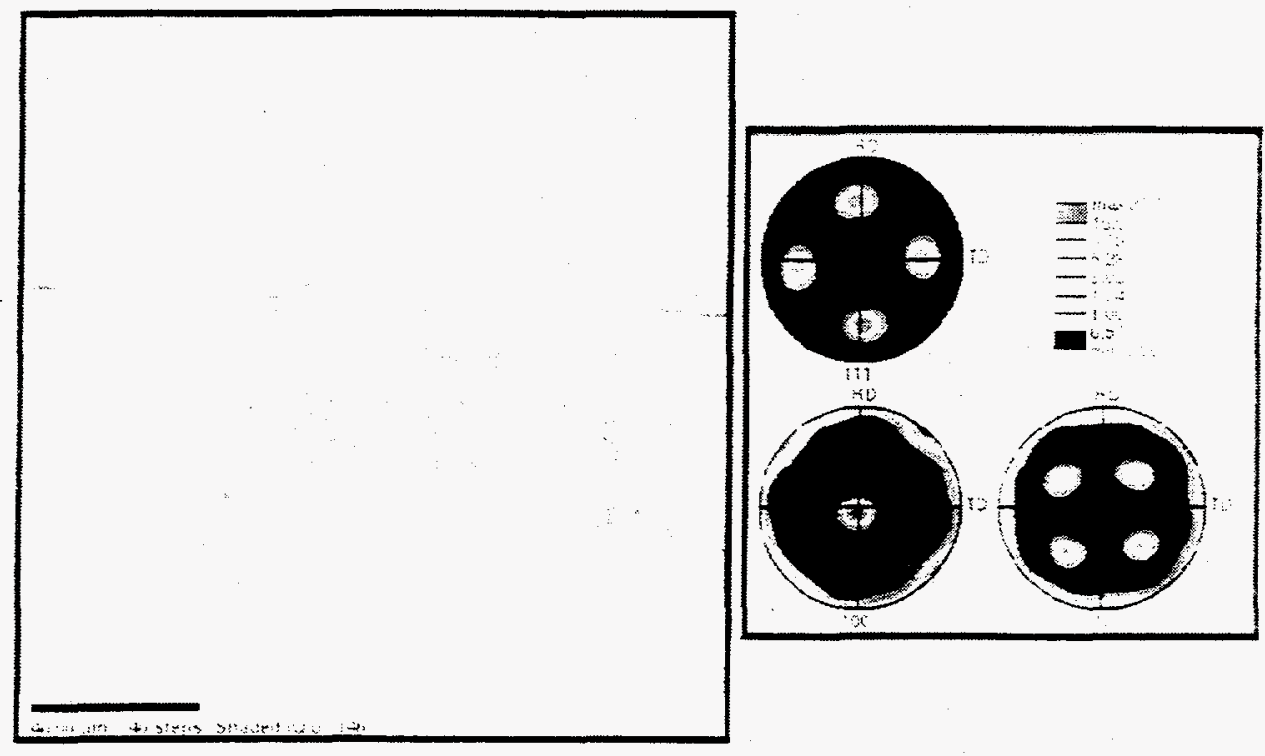

FIGURE 5. (a) Orientation image micrograph from a macroscopic region of a RABiT substrate of configuration $\left.\left.\mathrm{CeO}_{2}<110\right\rangle \| \mathrm{Ni}<100\right\rangle$. BKD patterns were obtained in a hexagonal grid with a step size of $1 \mu \mathrm{m}$. Variations in intensity are a reflection of pattern quality or intensity of the Kikuchi bands; (b) (111), (100), (110) pole figures of the orientations used to construct Figure 5 (a). 
In order to grow high quality epitaxial device films on the biaxially textured $\mathrm{Ni}$ substrate, a chemical and structural buffer layer is required. Typically, the desired buffer layers for superconductor film growth are oxides. Hence the task of fabricating a suitable substrate for epitaxial deposition of the superconductor involved epitaxial deposition of oxide buffer layers on $\mathrm{Ni}$. This is difficult because of the ease of surface oxide formation on $\mathrm{Ni}$ under the typical oxidizing conditions required for oxide film growth. Although the surface oxide on (100) $\mathrm{Ni}$ can be epitaxial, it typically forms a (111) textured $\mathrm{NiO}$ layer with three equivalent epitaxial relationships, resulting in many high angle boundaries. We have found two methods so far that have proven successful in producing a single orientation, cubeon-cube epitaxial oxide buffer layer films on rolled and recrystallized $\mathrm{Ni}$. The first involves epitaxial deposition of noble metal layers on $\mathrm{Ni}$ followed by deposition of oxides (19) and the second involves deposition of oxides directly on $\mathrm{Ni}$ under reducing conditions (20). A recent overview article summarizes the work on fabricating epitaxial superconductors using the RABiTS technique (21).

Oxide buffer layers can also be used for growth of epitaxial semiconductors. Figure 3 shows several possible multilayer structures that could be of use in the growth of epitaxial semiconductors like $\mathrm{Si}$ or GaAs. These are as follows: $\mathrm{CeO} \Omega \mathrm{Ni}, \mathrm{YSZ} / \mathrm{CeO}_{2} / \mathrm{Ni}, \mathrm{MgO} / \mathrm{Ag} / \mathrm{Pd} / \mathrm{Ni}$. Figure 4 shows $\mathrm{X}$-ray phi scans of the corresponding multilayer structures. Such structures have been grown using laser

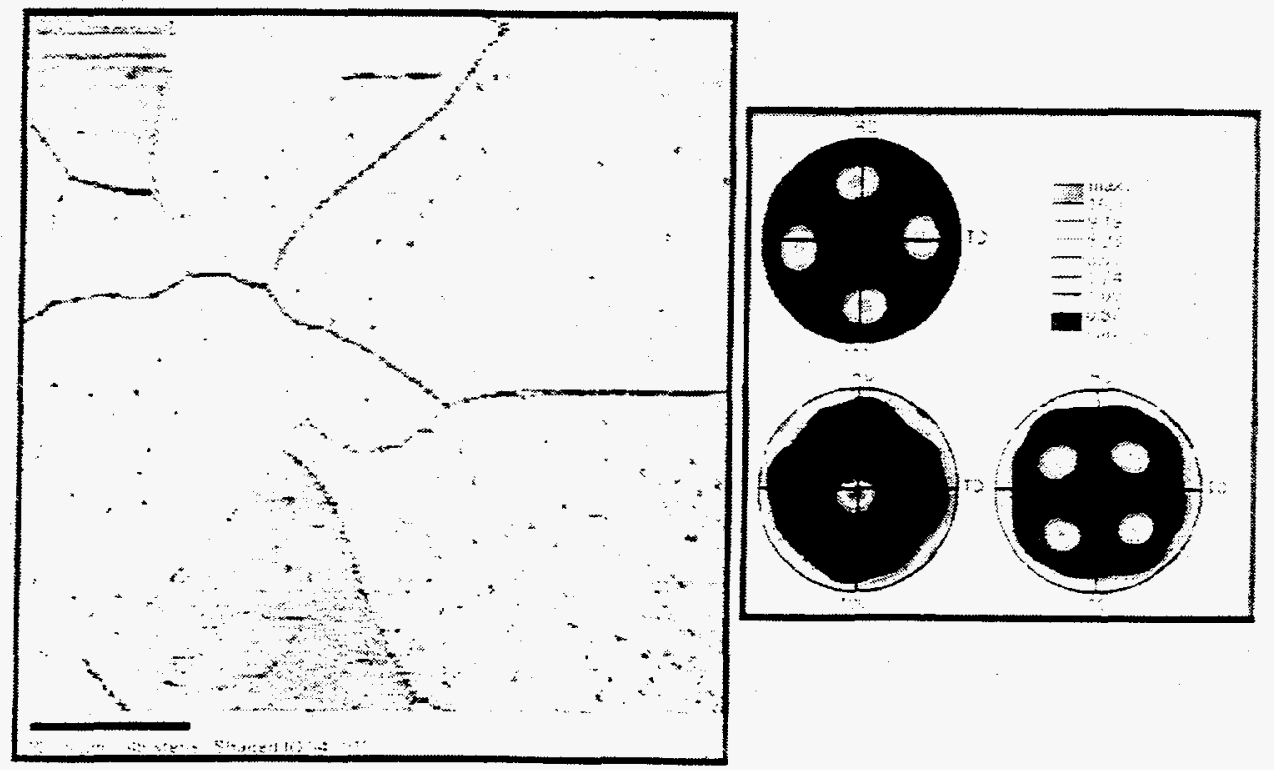

FIGURE 6. (a) Orientation image micrograph from a macroscopic region of a RABiT substrate of configuration $\left.\left.\mathrm{YSZ}<110\rangle \| \mathrm{CeO}_{2}<110\right\rangle \| \mathrm{Ni}<100\right\rangle$. BKD patterns were obtained in a hexagonal grid with a step size of $0.75 \mu \mathrm{m}$. Variations in intensity are a reflection of pattery quality or intensity of the Kikuchi bands; (b) (111), (100), (110) pole figures of the orientations used to construct Figure 6 (a). 

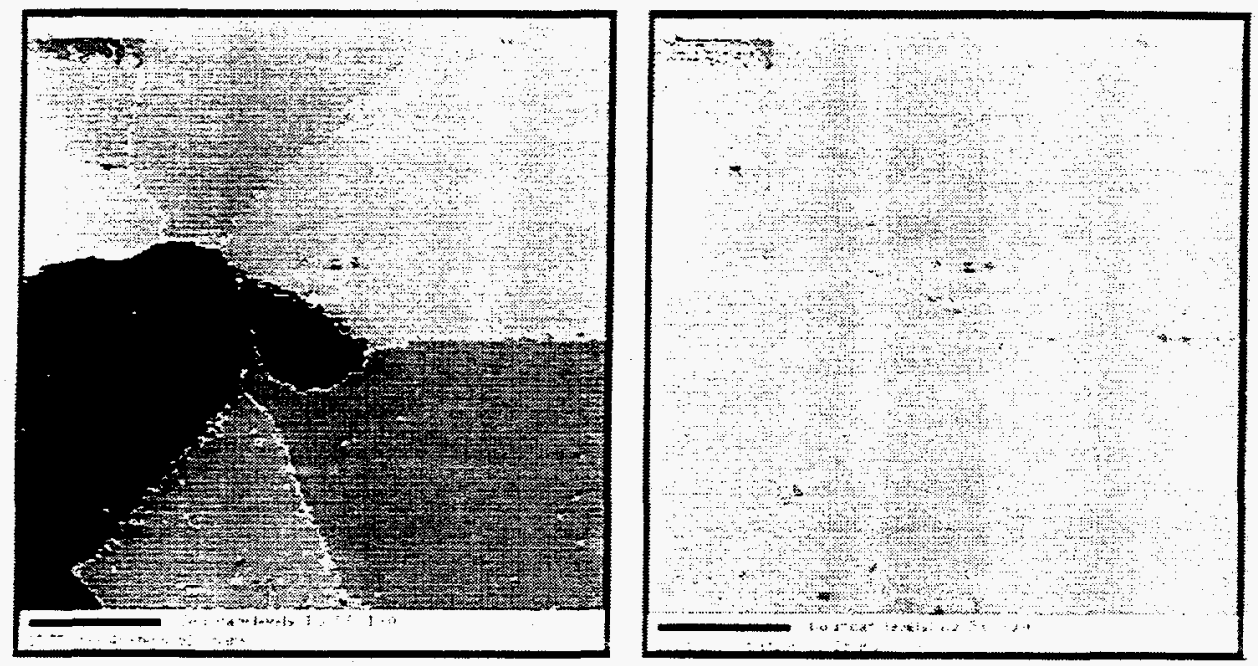

FIGURE 7. Orientation image micrographs shown in Figure $6 a$, shaded with the criterion that a given color represents a percolative region within (a) $2^{\circ}$ and (b) $5^{\circ}$. Clearly most of the substrate is well connected by boundaries less than $5^{\circ}$.

ablation (19-21), electron beam evaporation (22) and sputtering (23). Structures grown by laser ablation so far have resulted in evenly spaced cracks in the $\mathrm{CeO}$, layer (21). However, by controlling the deposition conditions and thickness of the $\mathrm{CeO}_{2}$ layer, crack free buffer layers have been obtained. Figure $5 \mathrm{a}$ shows an orientation image micrograph of a $\mathrm{CeO}_{2}$ layer approximately $175 \mathrm{~nm}$ thick on $\mathrm{Ni}$, grown using electron beam evaporation. Grain boundaries in the Ni substrate below are transferred through the oxide layers and are discerned in the orientation image since the pattern intensity from the boundary layer is poor. Figure 5 b shows the corresponding (111), (100) and (110) pole figures from the data in Figure 5a. Single orientation cube-on-cube epitaxy, with a rotation of $45^{\circ}$ with respect to the $\mathrm{Ni}$ substrate is obtained. Figure $6 \mathrm{a}$ shows an orientation image from the YSZ layer in a configuration shown in Figure 4 . Corresponding pole figures from this region are shown in Figure 6b. A single epitaxial orientation with a cube-on-cube with respect to the $\mathrm{CeO}_{2}$ layer is observed. If the region shown in Figure $6 \mathrm{a}$ is colored according to a criterion that a given color represents a percolative region of $2^{\circ}$ and $5^{\circ}$, then the corresponding images are shown in Figure $7 \mathrm{a}$ and $\mathrm{b}$ respectively. The entire region is of a single color in Figure $7 \mathrm{~b}$, indicating the near single crystal nature of the oxide surface. Chemical analysis of the buffer layers using electron microscopy indicates that for a deposition temperature of $780^{\circ} \mathrm{C}$, there is little diffusion of $\mathrm{Ni}$ into the $\mathrm{CeO}_{2}$ layer. 


\section{EPITAXIAL GROWTH OF SEMICONDUCTORS ON OXIDES}

Table 1 shows a compliation of epitaxial studies of oxides on Si (24-40). It is likely that similiar epitaxial relationships can be expected for growth of $\mathrm{Si}$ on

Table 1. Epitaxially grown oxides on Si.

\begin{tabular}{|c|c|c|c|c|c|}
\hline Material & $\begin{array}{c}\text { Out-of- } \\
\text { Plane } \\
\text { Orientation }\end{array}$ & $\begin{array}{c}\text { In-Plane } \\
\text { Orientation }\end{array}$ & Method & $\begin{array}{l}\text { Lattice } \\
\text { Mismatch }\end{array}$ & Ref. \\
\hline \multirow[t]{3}{*}{ YSZ } & $(001) \|(001)$ & {$[100] 11[100]$} & EB, PLD & $-5.8 \%$ & (25) \\
\hline & & & & & (26) \\
\hline & $(111) \|(111)$ & {$[110] \|[110]$} & PLD & $-5.8 \%$ & (27) \\
\hline $\mathrm{PrO}_{2}$ & $(111) \|(111)$ & {$[110] \mid 1[110]$} & PLD & $0.7 \%$ & (28) \\
\hline \multirow[t]{2}{*}{$\mathrm{CeO}_{2}$} & $(111) \|(111)$ & {$[110] \|[110]$} & $\mathrm{EB}$ & $0.4 \%$ & (29) \\
\hline & $(110) \|(001)$ & $[100] \|<100\rangle$ & EB & $0.4 \%$ & (30) \\
\hline \multirow[t]{2}{*}{$\mathrm{Y}_{2} \mathrm{O}_{3}$} & $(111) \|(111)$ & {$[110] \cup[110]$} & $E B$ & $-2.4 \%$ & (31) \\
\hline & $(110) \|(001)$ & {$[100] \| 1<100>$} & $\mathrm{EB}$ & $-2.4 \%$ & $(32)$ \\
\hline $\mathrm{MgAl}_{2} \mathrm{O}_{4}$ & $(001) \|(001)$ & {$[100] \|[100]$} & CVD & $0.7 \%$ & (33) \\
\hline \multirow[t]{2}{*}{$\mathrm{g}-\mathrm{Al}_{2} \mathrm{O}_{3}$} & $(001) \|(001)$ & {$[100] \|[100]$} & MOMBE & $-2.4 \%$ & (34) \\
\hline & $(111) \|(111)$ & {$[112] \|[112]$} & MOMBE & $-2.4 \%$ & (34) \\
\hline $\mathrm{MgO}$ & $(001) \|(001)$ & {$[100] 11[100]$} & PLD & $3.4 \%$ & (35) \\
\hline \multirow[t]{2}{*}{ Sro } & $(001) \|(001)$ & {$[100]||[100]$} & MBE & $-5.7 \%$ & $(36)$ \\
\hline & $(111) \|(111)$ & {$[112] \|[112]$} & MBE & $-5.7 \%$ & $(36)$ \\
\hline$\left(\mathrm{Sr}_{x} \mathrm{Ba}_{1-x} \mathrm{O}\right)$ & $(111) \|(111)$ & {$[112] \cup[112]$} & MBE & $-5.7-1 / 6 \%$ & (37) \\
\hline $\mathrm{BaO}$ & $(001) \|(001)$ & {$[100] 11[100]$} & MBE & $1.6 \%$ & (38) \\
\hline $\mathrm{SrTiO}_{3}$ & $(001) \| 1(001)$ & {$[110] 11[100]$} & FEB & $1.7 \%$ & (39) \\
\hline $\mathrm{LiNbO}_{3}$ & $(001) \|(111)$ & - & Sputtering & - & $(40)$ \\
\hline
\end{tabular}

Note: $\mathrm{EB}=$ electron beam deposition; $\mathrm{PLD}=$ pulsed laser deposition; $\mathrm{CVD}=$ chemical vapor deposition; (F)EB = (focussed) electron beam deposition; $(\mathrm{MO}) \mathrm{MBE}=$ (metaloragnic) molecular beam epitaxy.

oxides. The table shows the crystallographies. method of fabrication and lattice mismatch. The lattice mismatch refers to the near coincident site lattice mismatch for each epitaxial relationship. As reflected in the table, the three surfaces shown in Figure 2 may be well suited for growth of $\mathrm{Si}$. Other surfaces like $\mathrm{BaO}, \mathrm{SrTiO}_{3}$ etc could also be grown on RABiTS.

A key problem encountered in the growth of oxides on semiconductors has been that of oxidation of the semiconductor surface. This is a problem similar to that encountered during growth of oxides on $\mathrm{Ni}$. In the case of $\mathrm{Ni}$, formation of $\mathrm{NiO}$ is prevented by either depositing intermediate noble metal layers, or 
performing the deposition under a reducing $4 \% \mathrm{H}_{2}-96 \% \mathrm{Ar}$ background. This may also be possible during the growth semiconductors on some oxide surfaces, like YSZ and $\mathrm{CeO}_{2}$. On the other hand, the problem of $\mathrm{Si}$ oxidation during growth of $\mathrm{BaTiO}_{3}$ was overcome by depositing a thin layer of $\mathrm{BaO}$, which is stable against reduction by $\mathrm{Si}(38)$. Similar results have also been obtained if $\mathrm{BaO}$ is substituted with $\mathrm{MgO}$ (35) or SrO (39). Depending on the type of oxide, different approaches involving multilayer structures are possible. For example it is possible to grow $\mathrm{BaTiO}_{3} / \mathrm{Ge} / \mathrm{Si}(100)$ but not $\mathrm{BaTiO}_{3} / \mathrm{Si}(48)$. This is because of the differences in free energy of formation of $\mathrm{SiO}_{2}\left(-167 \mathrm{kcal} / \mathrm{mol}, 1000^{\circ} \mathrm{C}\right)$ and $\mathrm{GeO}_{2}(-90 \mathrm{kcal} / \mathrm{mol}$, $1000^{\circ} \mathrm{C}$ ) compared to that of decomposition of $\mathrm{BaTiO}_{3}$.

Table 2 lists a compilation of epitaxial oxides on GaAs. Clearly, the most studied oxide is $\mathrm{MgO}$ which has a very small lattice mismatch with GaAs. Cubeon-cube orientation of $\mathrm{MgO}$ on $\mathrm{GaAs}$ has also been observed as indicated in the table. Cube-on-cube has also been observed for $\mathrm{YSZ}$ and $\mathrm{In}_{2} \mathrm{O}_{3}$.

Table 2. Compliation of Epitaxial Oxides on GaAs.

\begin{tabular}{|l|l|l|l|l|l|}
\hline Material & $\begin{array}{c}\text { Out-of- } \\
\text { Plane } \\
\text { Orientation }\end{array}$ & $\begin{array}{c}\text { In-Plane } \\
\text { Orientation }\end{array}$ & Method & $\begin{array}{c}\text { Lattice } \\
\text { Mismatch }\end{array}$ & Ref. \\
\hline $\mathrm{YSZ}$ & $(001) \|(001)$ & {$[100] \|[100]$} & PLD & $-9.5 \%$ & {$[41]$} \\
\hline $\mathrm{In} 2 \mathrm{O}_{3}$ & $(001) \|(001)$ & {$[100] \|[100]$} & PLD & $0.6 \%$ & {$[42]$} \\
\hline $\mathrm{MgO}$ & $(001) \|(001)$ & {$[100] \|[100]$} & PLD & $-0.65 \%$ & {$[41]$} \\
\hline $\mathrm{MgO}$ & $(110) \|(001)$ & {$[110] \|[110]$} & $\mathrm{EB}$ & $-0.65 \%$ & {$[43]$} \\
\hline $\mathrm{MgO}$ & $(111) \|(001)$ & {$[110] \|[110]$} & PLD & $-0.65 \%$ & {$[42]$} \\
\hline $\mathrm{MgO}$ & $(111) \|(111)$ & {$[110] \|[110]$} & PLD & $-0.65 \%$ & {$[45]$} \\
\hline $\mathrm{PLZT}$ & $(110) \|(001)$ & - & Sputtering & $2.06-27.8 \%$ & {$[46]$} \\
\hline $\mathrm{LiNbO} 3$ & $(001) \|(111)$ & {$[110] \|[211]$} & PLD & $-0.86 \%$ & {$[47]$} \\
\hline
\end{tabular}

\section{EFFECT OF INTRAGRAIN DEFECTS ON PHOTOVOLTAIC PROPERTIES IN EPITAXIAL, MULTILAYER DEVICES}

Epitaxially grown multilayer semiconductor thin films can have a large concentration of defects due to lattice mismatches and thermal expansion mismatches between layers and also due to growth processes. Surface defects on the substrate or underlayer film can also extend into subsequently deposited layers, the most common of these being dislocations. Dislocations, like grain boundaries, provide charged deformation regions for carrier scattering. Another intragrain defect is the stacking fault, a planar defect. In a study of epitaxial growth of GaAs on Si single crystals, dislocation densities were controllably manipulated by Vemon et al. (49). They find that a dislocation density less than $5 \times 10^{6} / \mathrm{cm}^{2}$ is required to achieve a $20 \%$ efficiency. As the dislocation density increased to $10^{7} / \mathrm{cm}^{2}$, the open-circuit voltage, the short circuit current and the fill-factor decreased from their ideal values by $14 \%, 5 \%$ and $9 \%$ respectively (49). Kurtz and McConnel have 
summarized these results and based on data for efficiency versus dislocation densities and lifetime versus dislocation densities, have shown that a minority carrier lifetime $>10 \mathrm{~ns}$ is required to achieve $20 \%$ efficiency in GaAs (3). Hence in order to fabricate high efficiency devices on RABiTS, innovative multilayer sequences need to be grown to realize an intargranular defect density less than $10 \% \mathrm{~cm}^{2}$. This is perhaps the most challenging task for this approach of device fabrication. Kurtz and McConnel point out that besides GB recombination, perimeter recombination is also important and dominates the loss mechanism in single crystal films (3).

Since thin-films have a very high surface-to-volume ratios, when the thickness of the film becomes comparable to the mean free path of the carriers, the scattering of electrons and holes from the film surface can have a significant effects on transport properties (10). If the scattering process is elastic, i.e. specular reflection from the surface where only the velocity component perpendicular to the substrate is reversed, the energy remains constant and there is no loss. However, if after scattering, the carriers emerge from the surface with velocites independent of their incident ones, a change in momentum that occurs is reflected in the conductivity. This is known as diffuse or inelastic scattering. Since real surfaces are associated with some atomic disorder, inelastic scattering dominates. Hence surface passivation is normally employed. In polycrystalline films, GB passivation may also be required depending on the nature of the GBs.

\section{PASSIVATION OF SURFACE, INTERGRAIN AND INTRAGRAIN DEFECTS}

If one assumes that the primary effect of the defect and surface is intrinsic in nature, then passivation involves mitigation of dangling and distorted or dilated bonds. With respect to dangling bonds, heat-treating in hydrogen can be effective in hydrogenating these bonds in both $\mathrm{Si}(50-52)$ and $\mathrm{GaAs}$ (53). Similar results have been observed with $\mathrm{P}$ implanted in poly-Si (54). With respect to distorted or dilated bonds, the solution may be to replace the atoms at the GB with atoms forming stronger covalent bonds, e.g. $\mathrm{C}$ in $\mathrm{Si}$ and $\mathrm{P}$ in GaAs (10). This could be viewed as cladding the GB with a higher bandgap material or, alternatively, strengthening the distorted bond. Elements forming strong covalent bonds are, for example, H, B, C, P, and S. Such passivation can be performed either by post heat-treatment or by doping and subsequent segregation of impurities at grain boundaries. For free surfaces, $\mathrm{S}, \mathrm{Se}$ and $\mathrm{N}$ treatments have been effective in passivation (3). Higher bandgap layers also passivate the surface (3). Surface passivation can ce expected to be easier than grain boundary passivation, since the normal lattice density and bonding configurations are preserved at the GB, in contrast to severe structural perturbations present at free surfaces.

Not many studies reporting the passivation of intragrain defects, low-angle GBs and CSL GBs have been performed. During commercial growth of single crystal $\mathrm{Si}$, it appears that passivation of intragrain defects and very low-angle GBs is routine and simple to achieve (55). It is not however readily apparent why passivation of such low energy surfaces would be simple to achieve, since low 
energy boundaries would have less void space associated with them. In the presence of high energy interfaces, segregation of impurities and dopants to low energy boundaries may be restricted. However, this may not be the case when only low energy interfaces are present. Clearly, more detailed studies examining whether passivation of low-angle GBs and dislocations can be performed easily are required. Should this be the case, then epitaxial semiconductor films on RABiT substrates may be an ideal route to a high-efficiency, solar cell.

\section{SUMMARY}

In order to sustain and further the application of photovoltaic materials, devices with high efficiencies are required. Since cost is a major issue. thin film devices have considerable advantages over bulk materials. In case of thin films, choice of the substrate material dictates the performance of the device. For epitaxial films on single crystal substrates, very high efficiencies are obtained. However their cost precludes their use for large-scale applications. Amorphous and polycrystalline substrates with no controlled crystallographic texture and grain boundary character distribution, result in films with many grain boundaries. Since the grain size is difficult to increase to levels above where the effect of grain boundaries is negligible, the grain boundaries in these polycrystailine films reduce the effciencies to low levels. A review of studies relating the effect of grain boundaries on photovoltaic materials, suggests that control of grain boundaries may be one way to obtain high-effciency cells. Furthermore, anisotropy of growth rates and diffusion coefficients of impurity and dopant elements along differnt crystallographic directions, suggest that biaxial texture in these films and hence in the substrate may be desirable. An ideal substrate would be one that closely resembles a large single crystal. RABiT substrates formed by epitaxial growth of oxides on biaxially textured metals offer a potential route to a low cost, high efficiency cell. Such substrates have grains larger than $100 \mu \mathrm{m}$ and contain primarily low-angle grain boundaries, less than $5^{\circ}$. A review of grain boundaries studies in semiconductor materials suggests that this may be a cross-over point from low to high for photovoltaic properties. The substrates also have a very sharp crystallographic texture in all directions. A summary of studies relating to epitaxial growth of oxides on $\mathrm{Si}$ and $\mathrm{GaAs}$ was also included. Several commonly studied oxides which have been epitaxially deposited on these semiconductors, have also been deposited on RABiTS. Innovative sequencing of multilayers will however be required to ensure a low intragrain defect density in the photovoltaic film by matching lattice constants, thermal expansions etc. of the film with the substrate.

\section{ACKNOWLEDGEMENTS}

Research sponsored by U.S. Department of Energy, Office of Efficiency and Renewable Energy, Office of Utility Technology - Superconductivity Program and the Office of Energy Research, Basic Energy Sciences, managed by LockheedMartin Energy Research Corporation for the U.S. Department of Energy under contract DE-AC05-960R22464. 


\section{REFERENCES}

1. NREL News release, NR-01296, May 10. (1996), http://www.nrel.gov/hotstuff/press/thinfilm.html.

2. Zweibel, K., "Thin Films: Past, Present, Future," Progress in PV, The future of Thin Film Solar Cells, V. 3, \# 5, 279-294, (1995); Zweibel, K. and Kline, D., "Flat-Plate, Thin Film PV Systems", preprint, (1997).

3. Kurtz, S. and McConnell, R., "Requirements for a 20\%-Efficient Polycrystalline GaAs Solar Cell", preprint, this conference (1997).

4. Venkatasubramanian, R., O'Quinn, B. C., Hills, J. S., Sharps, P. R., Timmons, M. L., Hutchby J. A., Field, H., Ahrenkiel, R. and Keyes, B., "18.2\% efficient GaAs Solar Cell on Optical-grade Polycrystalline Ge Substrates", in Proceedings of the 25th IEEE Photovoltaic Specialists Conference, 1996, 31-36.

5. Bhat, R., Caneau, C., Zalt, C. E., Koza, M. A., Borner, W. A., Hwang, D. M., Schartz, S. A., Menocal S. G. and Favire, F. G., "Orientation dependence of S, Zn, Si, Te, and Sn doping in OMCVD growth of InP and GaAs - application to DH lasers and lateral p-n junction arrays grown on non-planar substrates," J. Cryst. Growth, 107, 772-778

6. Kondo, M., Anayama, C., Okada, N., Sekiguchi, H., Domen, K. and Tanabashi, T., "Crstallographic orientation dependence of impurity incorporation into III-IV compound semiconductors grown by metallorganic vapor phase epitaxy," J. Appl. Phys., 76. 914-927 (1994).

7. Pavesi, I., Piazza, F., Hernioi, M and Harrison, I., Orientation dependence of the Si doping of GaAs grown by molecular beam epitaxy." Semicond. Sci. and Tech., 8, 167-171 (1993).

8. Jones, S. H., Salinas, L. S., Jones, J. R. and Mayer, K, "Crystallographic orientation dependence of the growth rate for GaAs low pressure organometallic vapor phase epitaxy," J. of Electron. Mater., 24, 5-14 (1995).

9. Salermo, J. P., Fan, C. C., McClelland, R. W., Vohl, P., Mavroides, J. G., Bozler. C. O., "Electronic Properties of Grain Boundaries in GaAs: A Study of Oriented Bicrystals Prepared by Epitaxial Lateral Overgrowth," MIT Technical Repor 669, May 10, 1984.

10. "Polycrystalline and Amorphous Thin Fims and Devices", edited by Kazmerski, L. L., Academic Press, New York, 1980.

11. Bourret, A. and Desseaux, J., Phil. Mag., A39, 405 (1979); Bourret, A. and Desseaux, J., A39, 413 (1979).

12. Carter, C. B., Foll. H., Ast, D. G., Sass, S. L., Phil. Mag., A43, 441 (1981); Carter, C. B., Rose, J., Ast. D. G., Inst. Phys. Conf. Ser., 60, 153 (1981).

13. Matukura. Y. and Tanaka, S., J. Phys. Soc. Japan, 16, 833 (1961).

14. Mueller, R. K., J. Appl. Phys., 32, 640 (1961).

15. "Grain Boundaries in Semiconductors," edited by Leamy, H. J., Pike, G. E. and Seager, C. H., North-Holland. New York, 1982.

16. Card. H. C. and Yang E. S., "Electronic Processes at Grain Boundaries in Polycrystalline Semiconductors Under Optical Illumination," IEEE Trans. Electron Devices, ED-24, 397 (1977).

17. Singh, R., Bhar, T. N., Shewchun, J. and Loferski, J. J., "Effect of Grain Boundaries on the Performance of Polycrystalline Tunnel MIS Solar Cells," J. Vac. Sci. \& Tech., 16, 236 (1979). 
18. Kazmerski, L. L., "The Effects of Grain Boundaries and Interface recombination on the Performance of Thin-Film Solar Cells," Solid-State Electron, 21, 1545 (1978); Kazmerski, L. L., Sheldon, P. and Ireland, P. J., Thin-Solid Films. 58, 95 (1979).

19. Goyal, A, Norton, D. P., Budai, J. D., Paranthaman. M., Specht, E. D., Kroeger, D. M., Christen, D. K., He, Q., Saffian, B., List, F. A., Lee. D. F., Martin, P. M., Klabunde, C. E., Hatfied, E. and Sikka. V. K., "High critical Current Density Tapes by Epitaxial Deposition of $\mathrm{YBa}_{2} \mathrm{Cu}_{3} \mathrm{O}_{\mathrm{x}}$ Thick Films on Biaxially Textured Metals," Appl. Phys. Lett., Sept., 69, 1795 (1996).

20. Norton, D. P., Goyal, A., Budai, J. D., Christen, D. K., Kroeger, D. M., Specht, E. D., He. Q., Saffian, B., Paranthaman, M., Klabunde, C. E.. Lee, D. F., Sales, B. C., and List, F. A., "Epitaxial $\mathrm{YBa}_{2} \mathrm{Cu}_{3} \mathrm{O}_{\mathrm{x}}$ on Biaxially Textured Nickel (001): An approach to Superconducting Tapes with High Critical Current Density," Science. November, 274, 755 (1996).

21. Goyal, A, Norton. D. P., Kroeger, D. M., Christen. D. K., Paranthaman, M., Specht, E. D. Budai, J. D., He, Q., Saffian. B., List, F. A., Lee, D. F.. Hatfied, E., Klabunde, C. E. and Martin, P. M., "Conductors with Controlled Grain Boundaries: An Approach to the Next Generation, High temperature Superconducting Wire," To be published in the J. of Materials Research, Special 10th Anniversary Issue, November. (1997).

22. Paranthaman, M., Goyal, A, List, F. A., Specht, E. D., Lee, D. F., Martin, P. M., He, Q., Christen, D. K., Norton, D. P. and Budai, J. D., "Growth of Biaxially Textured Buffer Layers on Rolled-Ni Substrates by Electron Beam Evaporation." Physica C, 275, 266 (1997).

23. He. Q., Christen, D. K., Budai, J. D., Specht, E. D., Lee, D. F., Goyal, A, Norton, D. P., Paranthaman, M., List, F. A. and Kroeger, D. M., "Deposition of Biaxially-oriented Metal and Oxide Buffer-layer Films on Textured Ni Tapes: New Substrates for High-current, Hightemperature Superconductors," Physica C, 275, 155 (1997).

24. Fork, D. K. in Pulsed Laser Deposition of Thin Films, edited by Chrisey, D. B., and Hubler, G. H., John-Wiley and Sons, Inc., (1994).

25. Fukumoto, H., Imura, T. and Osaka, Y., Jpn. J. Appl. Phys. 27(8), L1404-L1405, (1988).

26. Fork, D. K., D. B. Fenner, G. A. N. Connell, et al. (1990), Appl. Phys. Lett. 57(11), 1137-1139.

27. Fork, D. K.. D. B. Fenner, R. W. Barton, et al. (1990). Appl. Phys. Lett. 57(11),11611163.

28. Fork, D. K., D. B. Fenner, and T. H. Geballe (1990). J. Appl. Phys. 68(8), 4316-4318.

29. Inoue, T., Y. Yamamoto, S. Koyama, et al. (1990). Appl. Phys. Lett. 56(14), 1332-1333.

30. Inoue, T., T. Ohsuna, L. Luo, et al. (1991), Appl. Phys. Lett. 59(27), 3604-3606.

31. Fukumoto, H., T. Imura, and Y. Osaka (1989), Appl. Phys. Lett. 55(4), 360-362.

32. Harada, K., H. Nakanishi, H. Itozaki. et al. (1991). Jpn. J. Appl. Phys. Lett. 30(5), 934-938.

33. Mikami, M., Y. Hokari, K. Egami, et al. (1983), Ext. Abstract, 15th Conference on Solid State Development and Materials, Tokyo, pp. 31-34.

34. Sawada, K., M. Ishida. T. Nakamura. et al. (1988), Appl. Phys. Lett. 52(20), 1672-1674.

35. Fork, D. K., F. Ponce, J. C. Tramontana, et al. (1991). Appl. Phys. Lett. 58(20), 2294 2296.

36. Kado, Y., and Y.Arita (1987), J. Appl. Phys. 61(6). 2398-2400.

37. Kado, Y., and Y. Arita (1988), Ext. Abstract, 20th Conference on Solid State Development and Materials, Tokyo, pp. 181-184.

38. McKee, R. A., F. J. Walker, and J. R. Conner, et al. (1991), Appl. Phys. Lett. 59(7), 782-784.

39. Mori, H., and H. Ishiwara (1991), Jpn. J. Appl. Phys. 30(8A), L1415-L1417. 
40. Rost, T. A., T. A. Rabson, B. A. Stone, et al. (1991), IEEE Trans. Ultrason., Ferroelectr. Freq. Control 38(6),640-643.

41. Nashimoto, K.. D. K. Fork, and T. H. Geballe (1992), Appl. Phys. Lett. 60(10), 1199-1201.

42. Tarsa, E. J., J. H. English and J. S. Speck (1993), Appl. Phys. Lett. 62(19), 2332-2334.

43. Huang, L. S., L.R. Zheng, and T. N. Blanton (1992), Appl. Phys. Lett 60(25), 3129-3131.

44. Tara, E. J., M. de Graef, D. R. Clarke, et al. (1993), J. Appl. Phys. 73(7), 3276-3282.

45. Fork, D. K., K. Nashimoto, and T. H. Geballe (1992), Appl. Phys. Lett. 60(13). 16211623.

46. Ishida, M., S. Tsuji, K. Kimura, et al. (1978), J. Cryst. Growth 45, 393-398.

47. Fork, D. K.. and G. B. Anderson (1993), Appl. Phys. Lett. 63(8), 1029-1031.

48. Jacobs, J. G.. Rho, Y. G., Pinizzotto, R. F., Proceedings of the MRS Symposium on Pulsed Laser Ablation, Dec. 1992, Boston, MA., 285, 379 (1993).

49. Vernon, S. M. and Tobin, S. P., "Experimental Study of Solar Cell Performance Versus Dislocation Density," Proceedings of tha 21st IEEE Photovoltaic Specialists Conference, 211 (1990).

50. Robinson. P. H. and D'Aiello, R. V., Appl. Phys., 39, 63 (1981).

51. Redfield, D., Appl. Phys. Lett., 38, 174 (1981).

52. Seager, C. S. and Ginley, D. S., J. Appl. Phys., 52, 1050 (1981).

53. Pearton, S. J. and Tavendale, A. J., J. Appl. Phys., 54, 1154 (1983).

54. Andrews, J. M., Electron. Mater. Conf., 20th, santa Barbara, California, June 28-30, (1978).

55. Solarex, private communication at conference on Future Generation PV Technologies. 